\title{
Effects of a Change in the Pattern of Insulin Delivery on Carbohydrate Tolerance in Diabetic and Nondiabetic Humans in the Presence of Differing Degrees of Insulin Resistance
}

\author{
Ananda Basu, ${ }^{\star}$ Aus Alzaid, ${ }^{\ddagger}$ Sean Dinneen, ${ }^{\star}$ Andrea Caumo, ${ }^{\S}$ Claudio Cobelli,, and Robert A. Rizza* \\ *Endocrine Research Unit, Mayo Clinic, Rochester, Minnesota 55905; ${ }^{\ddagger}$ Riyadh Armed Forces Hospital, Riyadh 11417, Saudi Arabia; ${ }^{\S}$ San \\ Raffaele Scientific Institute, Milan, Italy 20132; and ${ }^{\|}$Department of Electronics and Informatics, University of Padova, Padova, Italy 35131
}

\begin{abstract}
While it is well established that people with non-insulin dependent diabetes mellitus have defects in both insulin secretion and action, the relative contribution of each to glucose intolerance is not known. Therefore, nondiabetic (lean and obese) and non-insulin dependent diabetes mellitus subjects were studied on two occasions. On each occasion, insulin secretion was inhibited with somatostatin and glucose was infused in a pattern and amount that mimicked the systemic delivery rate normally observed after ingestion of $50 \mathrm{~g}$ of glucose. Insulin also was infused so as to mimic postprandial insulin profiles observed in separate groups of diabetic and nondiabetic subjects after food ingestion. Glucose turnover was measured using the isotope dilution method. A delayed pattern of insulin delivery (i.e., a "diabetic" insulin profile) led to higher $(P<0.05)$ glucose concentrations in all groups; however, the effects were transient, resulting in only a modest increase in the integrated glycemic responses. An isolated defect in insulin action had little effect on peak glucose concentration; however, it prolonged the duration of hyperglycemia, leading to a $2.5-4.2$-fold increase $(P<0.05)$ in the integrated glycemic response. A combined defect in the pattern of insulin secretion and action was additive rather than synergistic. Both defects caused hyperglycemia by altering suppression of endogenous glucose release and stimulation of glucose disposal. Whereas obese diabetic and nondiabetic subjects had comparable defects in glucose clearance, non-insulin dependent diabetes mellitus subjects also had defects in hepatic insulin action. Thus, abnormalities in the pattern of insulin secretion and action alone or in combination impair glucose tolerance. An isolated defect in insulin action has a more pronounced and prolonged effect than does an isolated change in the pattern of insulin secretion. Hepatic and extrahepatic insulin resistance results in marked and sustained hyperglycemia. (J. Clin. Invest. 1996. 97:2351-2361.) Key words: obesity • non-insulin dependent diabetes mellitus - postprandial • endogenous glucose release $\cdot$ glucose utilization
\end{abstract}

Address correspondence to R.A. Rizza, Endocrine Research Unit, Mayo Clinic, 200 First Street S.W., Rochester, MN 55905. Phone: 507255-6515; FAX: 507-255-4828.

Received for publication 4 December 1995 and accepted in revised form 28 February 1996.

J. Clin. Invest.

(C) The American Society for Clinical Investigation, Inc.

0021-9738/96/05/2351/11 \$2.00

Volume 97, Number 10, May 1996, 2351-2361

\section{Introduction}

Glucose concentrations in nondiabetic individuals rarely exceed $140-150 \mathrm{mg} / \mathrm{dl}$ following food ingestion (1-9). This is because even a slight increase in glucose produces a prompt increase in insulin (1-9). The coordinated rise in insulin and glucose suppresses endogenous glucose release and stimulates glucose uptake which, in turn, minimizes any further rise in glucose concentration (4-9). In contrast, carbohydrate ingestion results in marked and sustained hyperglycemia in people with diabetes (1-9). There are several possible reasons why this may occur. Insulin secretion is both decreased and delayed in non-insulin dependent diabetes mellitus (NIDDM) ${ }^{1}$ (1-9). Furthermore, people with NIDDM are resistant to insulin (10-14).

Numerous studies have demonstrated that either of these abnormalities have the potential to alter glucose tolerance (1-14). However, the relative contribution of each to abnormal postprandial glucose metabolism in NIDDM has not been established. In particular, it is not currently known whether defects in insulin secretion and insulin action result in the same pattern and degree of hyperglycemia, nor is it known whether the two together cause a greater impairment in carbohydrate tolerance than either alone. Such an outcome is not inevitable since hyperglycemia per se, by promoting glucose uptake and inhibiting glucose release (15-20), could compensate in part or in whole for a decrease in the pattern of insulin secretion or action. Novel therapies that improve insulin action or that restore the pattern and amount of circulating insulin to concentrations observed in nondiabetic subjects are in the process of being developed (21-23). Therefore, the answers to the above questions are of practical as well as physiologic interest.

In the present experiments, we sought to determine the relative contribution of defects in insulin secretion and action to postprandial carbohydrate tolerance. To do so, we first characterized the postprandial insulin profile observed in a typical group of people with NIDDM after eating a standard mixed meal. We then used a computer driven infusion system to deliver insulin so as to reproduce this "diabetic" profile as well as a "nondiabetic" insulin profile previously observed in healthy lean nondiabetic subjects (24). Endogenous insulin secretion was inhibited with somatostatin on both occasions. Glucose also was infused so as to mimic the amount and pattern of glucose that normally enters the systemic circulation following ingestion of $50 \mathrm{~g}$ of carbohydrate $(4,6,8)$. This experimental design enabled us to ascertain the effects of $(a)$ changes in insulin action by comparing the glycemic responses observed in the lean vs obese vs NIDDM subjects during a nondiabetic insulin profile, $(b)$ changes in the pattern of insulin secretion by com-

1. Abbreviation used in this paper: NIDDM, non-insulin dependent diabetes mellitus. 
paring the glycemic responses observed during the nondiabetic and diabetic insulin profiles in each of the three groups, and (c) changes in both the pattern of insulin secretion and action by comparing the glycemic responses observed during the diabetic insulin profile in the two insulin-resistant groups to those observed during the nondiabetic insulin profile in the lean insulin-sensitive subjects. Measurement of glucose turnover also enabled us to determine the relative contribution of changes in the pattern and rates of endogenous glucose release and disposal to the alterations in glucose tolerance.

\section{Methods}

Subjects. After approval from the Mayo Institutional Review Board, 7 lean nondiabetic, 13 obese nondiabetic, and 14 obese subjects with NIDDM gave informed written consent to participate in the study. Seven of the diabetic and six of the obese nondiabetic subjects participated in the mixed meal study in which the postprandial diabetic insulin profile was defined. The remainder of the subjects participated in the prandial glucose and insulin infusion studies in which the effects of alterations in the pattern of insulin delivery in the presence of differing degrees of insulin resistance were examined. The characteristics of the subjects are shown in Table I. Age and gender did not differ either between or within groups in the two studies. Body-mass index in the NIDDM and obese nondiabetic subjects also did not differ either between or within groups. However, the percent body fat was slightly lower $(P<0.05)$ in the NIDDM than the obese nondiabetic subjects. By design, the lean nondiabetic subjects had a lower bodymass index and a lower percentage of body fat than did the two obese groups. The mean glycosylated hemoglobin concentrations (GlycAffin; Isolab Inc., Akron, $\mathrm{OH}$; normal range 4-7\%) did not differ in the NIDDM subjects in the mixed meal and the prandial glucose and insulin infusion studies $(10.0 \pm 1.0$ vs $9.5 \pm 1 \%)$. At the time of screening, three of the diabetic subjects in the mixed meal studies and six in the prandial glucose and insulin infusion studies were taking sulphonylureas. These agents were discontinued at least $48 \mathrm{~h}$ before study. One diabetic subject in the mixed meal study was taking intermediate-acting insulin, which was discontinued $36 \mathrm{~h}$ before the study. All subjects were in good health, had normal blood pressure, and were at stable weight. None regularly engaged in vigorous exercise or took any medication other than sulfonylureas. The nondiabetic subjects did not have a history of diabetes in their first-degree relatives.

Experimental design. For the mixed meal studies, subjects were admitted to the General Clinical Research Center at 1700 hours on the evening before the study. A standard $10-\mathrm{cal} / \mathrm{kg}$ meal (50\% carbohydrate, $15 \%$ protein, and $35 \%$ fat) was eaten between 1730 and 1800 hours after which subjects were fasted. At $\sim 0600$ the next morning, an 18-gauge catheter was inserted retrogradely into a hand vein and this hand was placed in a heated Plexiglas box and maintained at a temperature of $\sim 55^{\circ} \mathrm{C}$ to allow sampling of arterialized blood. At
0800 , subjects ingested a mixed meal ( $472 \mathrm{kcal} ; 45 \%$ carbohydrate as $50 \mathrm{~g}$ of glucose contained in jello, and $40 \%$ fat and $15 \%$ protein as scrambled eggs). Blood was sampled at regular intervals before and after the meals to permit measurement of glucose and insulin concentrations.

For the prandial glucose and insulin infusion studies, subjects were admitted to the General Clinical Research Center the evening before study and an 18-gauge catheter was inserted into a forearm vein. After ingestion of a standard evening meal, the nondiabetic subjects were infused overnight with $0.9 \%$ saline; whereas, the diabetic subjects were infused with insulin. The insulin infusion was adjusted to maintain plasma glucose concentrations during the night at $\sim 5$ $\mathrm{mM}$ in the diabetic subjects $(20,25,26)$. On the following morning, an additional cannula was inserted retrogradely into a dorsum hand vein at 0600 . The hand was then placed in a heated Plexiglas box as in the mixed meal study and maintained at a temperature of $\sim 55^{\circ} \mathrm{C}$ to allow sampling of arterialized venous blood.

Primed-continuous infusions of $\left[{ }^{14} \mathrm{CO}_{2}\right]$ bicarbonate $(110 \mu \mathrm{Ci}$ prime, $1.1 \mu \mathrm{Ci} / \mathrm{min}$ continuous) and $\left[6-{ }^{3} \mathrm{H}\right]$ glucose $(17 \mu \mathrm{Ci}$ prime, 0.17 $(\mathrm{Ci} / \mathrm{min}$ continuous $)$ were started at $0700(-180 \mathrm{~min})$ and $0800(-120$ $\mathrm{min}$ ) hours, respectively, and continued until the end of the study. At 1000 (time zero), an infusion of somatostatin (60 ng/kg/per min) was started in all groups in order to inhibit endogenous hormone secretion. Growth hormone (3 ng/kg/per min), and glucagon $(0.65 \mathrm{ng} / \mathrm{kg} /$ per min for the first $2 \mathrm{~h}$ and $1.3 \mathrm{ng} / \mathrm{kg} /$ per min thereafter) also were infused to replace basal levels. On one occasion, insulin was infused in a manner designed to produce a nondiabetic postprandial insulin profile, mimicking that previously observed in lean nondiabetic subjects after glucose ingestion (24). On the other occasion, it was infused to produce a diabetic profile, mimicking the plasma insulin concentration observed in the diabetic subjects in the mixed meal study. The insulin infusion rates to produce the nondiabetic and diabetic profiles are given in Table II. The order of study was random. The diabetic profile used the identical amount of insulin as the nondiabetic profile, with the insulin infusion program being modified to create a lower peak at a later time (i.e., $120 \mathrm{~min}$ ).

Glucose was infused as previously described (20) in an amount $(\sim 35 \mathrm{~g})$ and pattern that mimicked the systemic rate of delivery of glucose that normally occurs following ingestion of $50 \mathrm{~g}$ of glucose (4, 6,8 ). To avoid the confounding effects introduced by counter regulation, the study was terminated when the glucose concentration fell below $4.3 \mathrm{mmol} /$ liter, at which time the intravenous infusions were discontinued and subjects were given glucose. In these instances, data generated thereafter (i.e., the last 2-3 time points) were excluded from analysis. Glucose levels fell below $4.3 \mathrm{mmol} /$ liter during both the nondiabetic and diabetic insulin profiles in four of the seven lean nondiabetic subjects. In contrast, glucose levels fell to below 4.3 $\mathrm{mmol} /$ liter in only one of the obese subjects during the diabetic profile and in none of the NIDDM subjects during either profile.

All infused glucose contained $\left[6-{ }^{3} \mathrm{H}\right]$ glucose. The basal infusion of $\left[6-{ }^{3} \mathrm{H}\right]$ glucose was varied to approximate the anticipated pattern of change of endogenous glucose release (20). By so doing, the mean

Table I. Characteristics of the Study Groups

\begin{tabular}{|c|c|c|c|c|c|}
\hline & \multicolumn{2}{|c|}{ Mixed meal study } & \multicolumn{3}{|c|}{ Insulin infusion studies } \\
\hline & $\begin{array}{c}\text { Obese } \\
\text { nondiabetic }\end{array}$ & NIDDM & $\begin{array}{c}\text { Lean } \\
\text { nondiabetic }\end{array}$ & $\begin{array}{c}\text { Obese } \\
\text { nondiabetic }\end{array}$ & NIDDM \\
\hline Age (yr) & $50 \pm 3$ & $53 \pm 3$ & $50 \pm 3$ & $50 \pm 4$ & $57 \pm 3$ \\
\hline Gender $(\mathrm{M} / \mathrm{F})$ & $4 / 2$ & $4 / 3$ & $4 / 3$ & $3 / 4$ & $5 / 2$ \\
\hline Body mass index* & $28 \pm 2$ & $31 \pm 2$ & $22 \pm 1$ & $34 \pm 2$ & $31 \pm 1$ \\
\hline Percentage of body fat & - & - & $26 \pm 3$ & $44 \pm 3$ & $32 \pm 3$ \\
\hline
\end{tabular}

*Weight in kilograms divided by square of height, in meters. 


\begin{tabular}{|c|c|c|c|c|c|c|c|c|c|c|c|c|c|c|c|}
\hline \multicolumn{16}{|c|}{ Milliunits per kilogram lean body mass per min } \\
\hline Time (min) & 0 & 10 & 20 & 30 & 40 & 50 & 60 & 70 & 80 & 90 & 100 & 110 & 120 & 130 & 140 \\
\hline Nondiabetic profile & 0.391 & 0.735 & 1.009 & 0.902 & 0.753 & 0.665 & 0.652 & 0.608 & 0.444 & 0.571 & 0.491 & 0.346 & 0.346 & 0.346 & 0.346 \\
\hline \multirow[t]{3}{*}{ Diabetic profile } & 0.325 & 0.351 & 0.379 & 0.377 & 0.436 & 0.47 & 0.494 & 0.508 & 0.521 & 0.529 & 0.532 & 0.533 & 0.527 & 0.516 & 0.507 \\
\hline & \multicolumn{15}{|c|}{ Milliunits per kilogram lean body mass per min } \\
\hline & 150 & 160 & 170 & 180 & 190 & 200 & 210 & 220 & 230 & 240 & 250 & 260 & 270 & 280 & \\
\hline Nondiabetic profile & 0.346 & 0.346 & 0.346 & 0.346 & 0.346 & 0.346 & 0.346 & 0.346 & 0.346 & 0.346 & 0.346 & 0.346 & 0.346 & 0.346 & \\
\hline Diabetic profile & 0.499 & 0.487 & 0.476 & 0.468 & 0.454 & 0.444 & 0.434 & 0.423 & 0.411 & 0.4 & 0.387 & 0.375 & 0.363 & 0.352 & \\
\hline
\end{tabular}

plasma glucose-specific activity was maintained within $5 \%$ of basal values throughout the study in all groups. Breath was collected for measurement of ${ }^{14} \mathrm{CO}_{2}$ specific activity as previously described (6). Subjects were requested to void just before and at the end of the study to enable measurement of urine glucose excretion.

Analytical technique. Arterialized plasma samples were placed in ice, centrifuged at $4^{\circ} \mathrm{C}$, separated, and stored at $-20^{\circ} \mathrm{C}$ until assay. $\mathrm{C}$-peptide and glucagon concentrations were measured using reagents purchased from Linco Research Inc. (St. Louis, MO). Growth hormone was measured using reagents purchased from ICN Biochemicals Inc. (Costa Mesa, CA). Glucose-specific activity was measured as previously described (4). Lean body mass and percentage of body fat were determined by dual-energy x-ray absorptiometry (DPX scanner; Lunar Corporation, Madison, WI). Glucose concentrations were measured using a glucose analyzer (Yellow Springs Instrument Co., Yellow Springs, $\mathrm{OH}$ ).

Calculation of glucose turnover. Glucose-specific activity was smoothed using the OOPSEG program of Bradley et al. (27). Glucose appearance and disappearance were calculated using the equations of Steele et al. (28). Glucose clearance was calculated by dividing glucose disappearance by glucose concentration. Endogenous glucose release was determined by subtracting the glucose infusion rate from the tracer-determined rate of glucose appearance. The percentage of glucose derived from ${ }^{14} \mathrm{CO}_{2}$ was calculated by dividing the plasma $\left[{ }^{14} \mathrm{C}\right]$ glucose-specific activity by breath ${ }^{14} \mathrm{CO}_{2}$-specific activity (6). Rates of glucose disappearance and clearance are expressed per kilogram lean body wt.

Statistical analysis. Data in the text and figures are expressed as mean \pm SEM. Integrated responses and responses above basal were calculated using the trapezoidal rule. Integrated response was defined as total area above zero during the insulin infusions. Area above basal was defined as the area above the mean of values present during the $30 \mathrm{~min}$ before the insulin infusions. Paired tests were used to compare results within groups (i.e., nondiabetic vs diabetic profiles). Analysis of variance was used to compare results among groups (lean vs obese vs NIDDM subjects) followed by nonpaired tests if significance was observed. Comparisons were made using either Student's or Windsorized $t$ tests (29). One-tailed tests were used to test the hypothesis that a decrease in the pattern of insulin secretion or action resulted in higher blood and urinary glucose concentrations, lower disposal, and greater endogenous glucose release. All other tests were two tailed. A $P<0.05$ was considered statistically significant.

\section{Results}

Glucose and insulin concentrations following mixed meal ingestion. Plasma glucose concentrations were higher $(P<0.01)$ in the obese NIDDM than in the obese nondiabetic subjects both before $(10.2 \pm 1.0 \mathrm{vs} 5.0 \pm 0.1 \mathrm{mmol} / \mathrm{liter})$ and after $(3.9 \pm 0.3 \mathrm{vs}$ $1.8 \pm 0.1 \mathrm{mmol} /$ liter per $5 \mathrm{~h}$ ) meal ingestion (Fig. 1). Plasma glucose concentrations peaked in the nondiabetic subjects at $\sim 9.0$ $\mathrm{mmol} /$ liter at $60 \mathrm{~min}$, returning to basal values by $\sim 120 \mathrm{~min}$. Preprandial insulin concentrations did not differ between groups. Insulin concentrations during the first $2 \mathrm{~h}$ after meal ingestion were lower $(P<0.01)$ in the diabetic than nondiabetic subjects $(7.8 \pm 3.1$ vs $32.5 \pm 7.8 \mathrm{nmol} /$ liter per $2 \mathrm{~h})$. Insulin concentrations did not differ between groups thereafter.

C-peptide, glucagon, and growth hormone concentrations during prandial glucose and insulin infusions. Somatostatin was infused to ensure that endogenous hormone secretion was inhibited in all groups. C-peptide concentrations at time zero (i.e., before the start of the somatostatin infusion) differed among groups $(P<0.01)$ being highest in the obese nondiabetic subjects, next highest in the lean nondiabetic subjects, and lowest in the obese NIDDM subjects (Fig. 2). Somatostatin promptly inhibited insulin secretion in all groups, resulting in C-peptide concentrations that did not differ in any of the three groups during either the nondiabetic or diabetic insulin profiles. Glucagon and growth hormone concentrations also did not differ among the three groups during the somatostatin/ replacement hormone infusions on either study day.
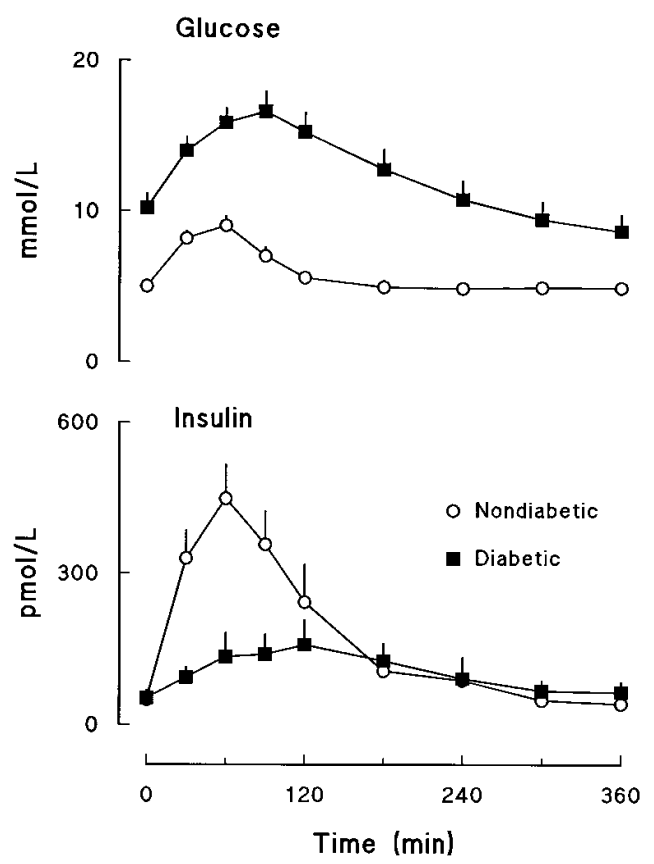

Figure 1. Glucose and insulin concentrations observed in obese NIDDM and nondiabetic subjects following ingestion of a mixed meal at time zero. 

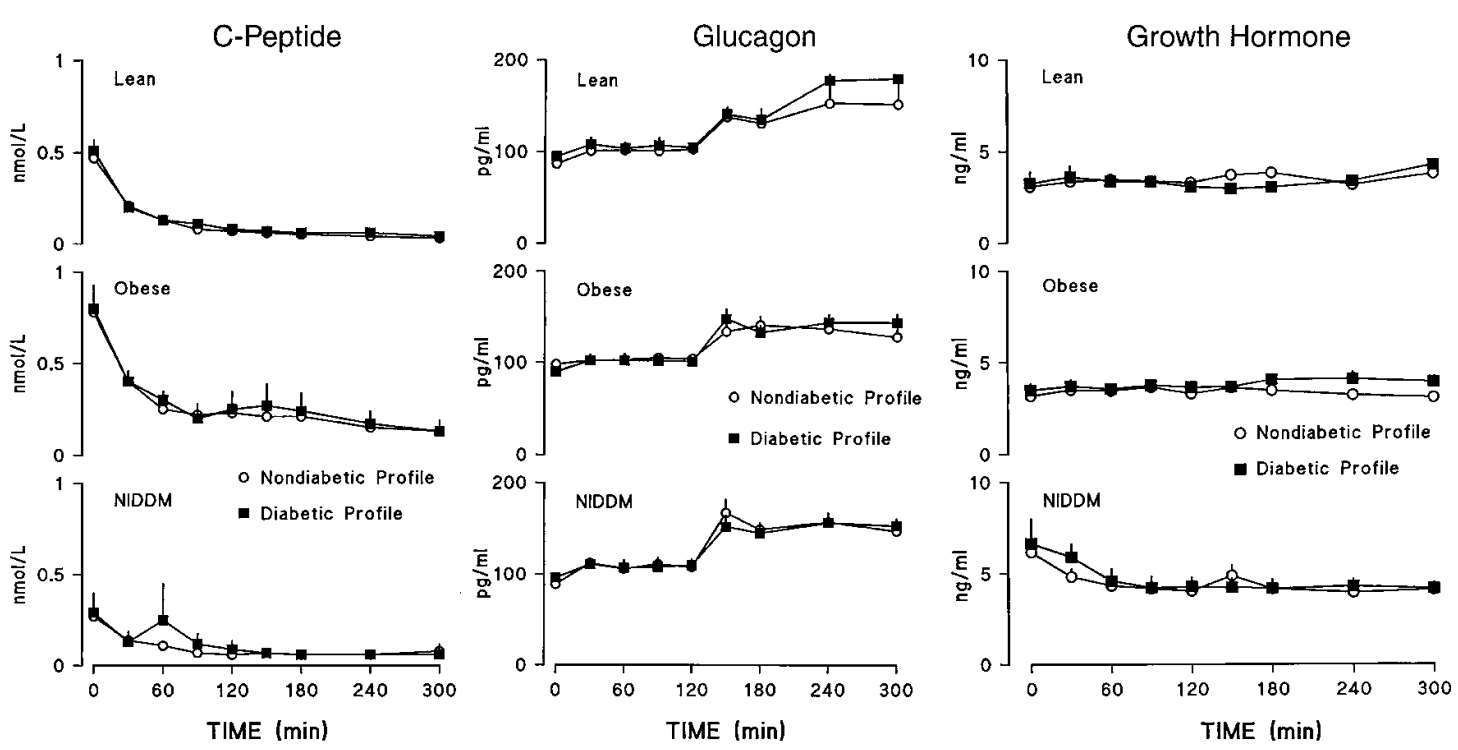

Figure 2. C-peptide (left), glucagon (middle), and growth hormone (right) concentrations observed in the lean nondiabetic, obese nondiabetic, and subjects with NIDDM during the nondiabetic and diabetic insulin profiles. An infusion of somatostatin, glucagon, and growth hormone was started at time zero.

\section{Effects of differences in insulin action on glucose tolerance}

Glucose and insulin concentrations in lean, obese, and NIDDM subjects. Glucose concentrations in the lean nondiabetic subjects increased to a peak of $\sim 9 \mathrm{mmol}$ at $40-50 \mathrm{~min}$ and returned to basal values by $\sim 120$ min during the nondiabetic insulin profile (Fig. 3, top left). This pattern was virtually superimposable on that observed in the nondiabetic subjects after meal ingestion (see Fig. 1, top), indicating that the prandial glucose infusion and the nondiabetic insulin profile modeled from previous data were sufficiently well matched to result in near normal glucose tolerance.

The NIDDM subjects were infused with insulin during the night so that glucose concentrations would be equal in all groups at the start of the study. This resulted in higher $(P<$ $0.05)$ basal insulin in the NIDDM subjects on both study days (156 \pm 75 , and $156 \pm 61 \mathrm{pmol} /$ liter), than those observed in the obese nondiabetic subjects $(58 \pm 8$, and $59 \pm 10 \mathrm{pmol} / \mathrm{liter})$ and which in turn were higher than those observed in the lean nondiabetic subjects $(33 \pm 4$, and $34 \pm 3 \mathrm{pmol} /$ liter $)$ consistent with varying degrees of insulin resistance (Fig. 3, right). Insulin concentrations observed during the diabetic insulin profile closely mimicked those observed in the NIDDM subjects following meal ingestion (see Fig. 1, bottom).

We anticipated that insulin action would differ among the three groups. This was confirmed by demonstrating that despite infusion of identical amounts of insulin and glucose, the
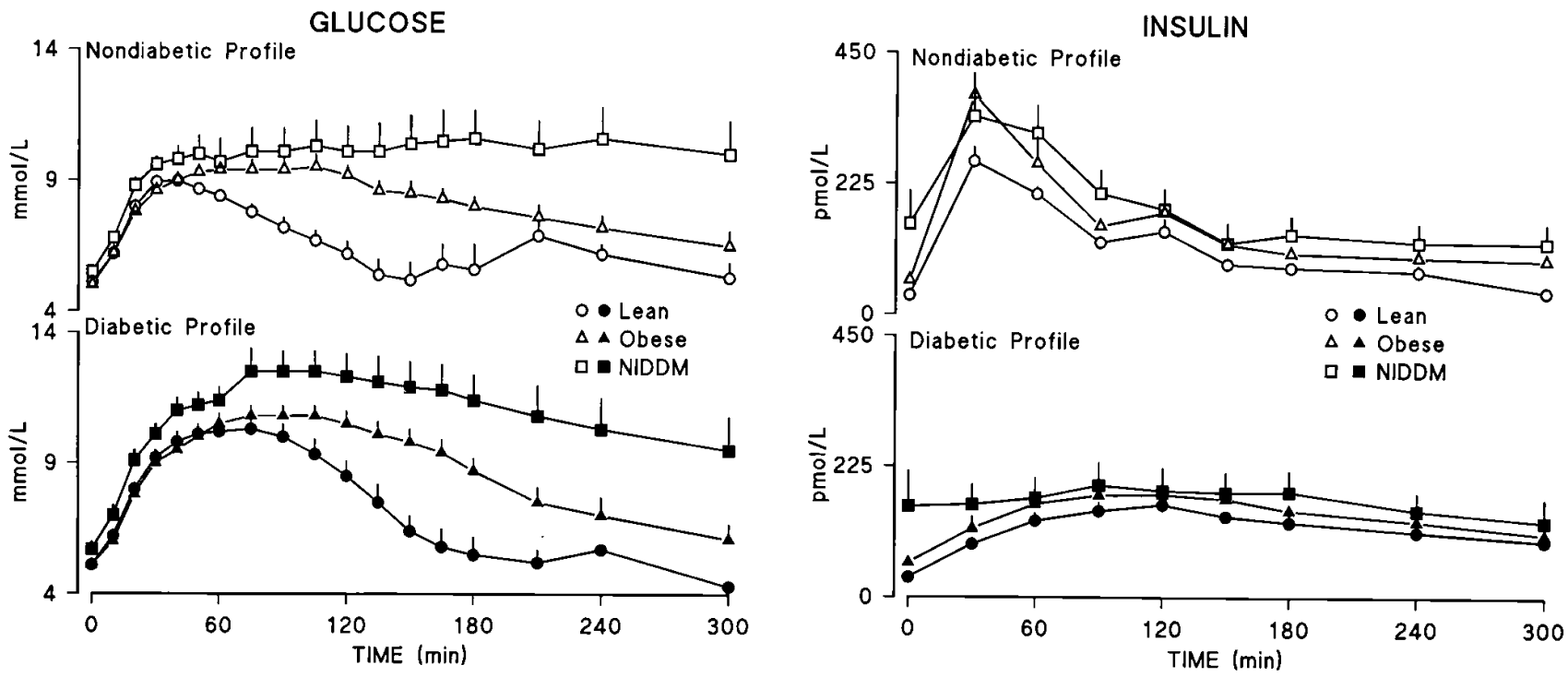

Figure 3. Glucose (left) and insulin (right) concentrations observed in the lean nondiabetic, obese nondiabetic, and NIDDM subjects during the nondiabetic and diabetic insulin profiles. 
$(P=0.04-0.06)$ in the NIDDM subjects $(13.0 \pm 1.0 \mathrm{mmol} / \mathrm{liter})$ than those observed in either the lean $(10.6 \pm 0.4 \mathrm{mmol} / \mathrm{liter})$ or obese $(11.0 \pm 0 \mathrm{mmol} /$ liter $)$ nondiabetic subjects.

Glucose turnover in lean, obese, and NIDDM subjects. To determine the mechanism by which different degrees of insulin action led to different degrees of glucose tolerance, rates of glucose disappearance and endogenous glucose release were measured. Basal rates of endogenous glucose release differed $(P<0.02)$ among groups on both study days (Fig. 4, top left). In each instance, basal endogenous glucose release was higher $(P<0.01)$ in the NIDDM than in the lean subjects. Despite the fact that basal insulin concentrations were two- to threefold higher in the NIDDM than obese nondiabetic subjects, basal endogenous glucose release did not differ between groups. Integrated endogenous glucose release was greater $(P<0.01)$ in the NIDDM subjects than in the obese subjects during both the nondiabetic and diabetic insulin profiles. Integrated endogenous glucose release was slightly, but significantly, $(P<0.05)$ higher in the obese than lean nondiabetic subjects. The rate of incorporation of carbon dioxide into glucose showed a similar pattern with the integrated response being greater $(P<0.01)$ in the NIDDM than obese subjects on both study days (Fig. 4, bottom left).

Glucose disappearance did not differ among groups either before or during the nondiabetic or diabetic insulin profiles (Fig. 4, top right). However, the rates of disappearance were not appropriate for the higher prevailing glucose concentrations in either the obese or NIDDM subjects. This was evident concentrations during the diabetic profile tended to be higher

ENDOGENOUS GLUCOSE RELEASE
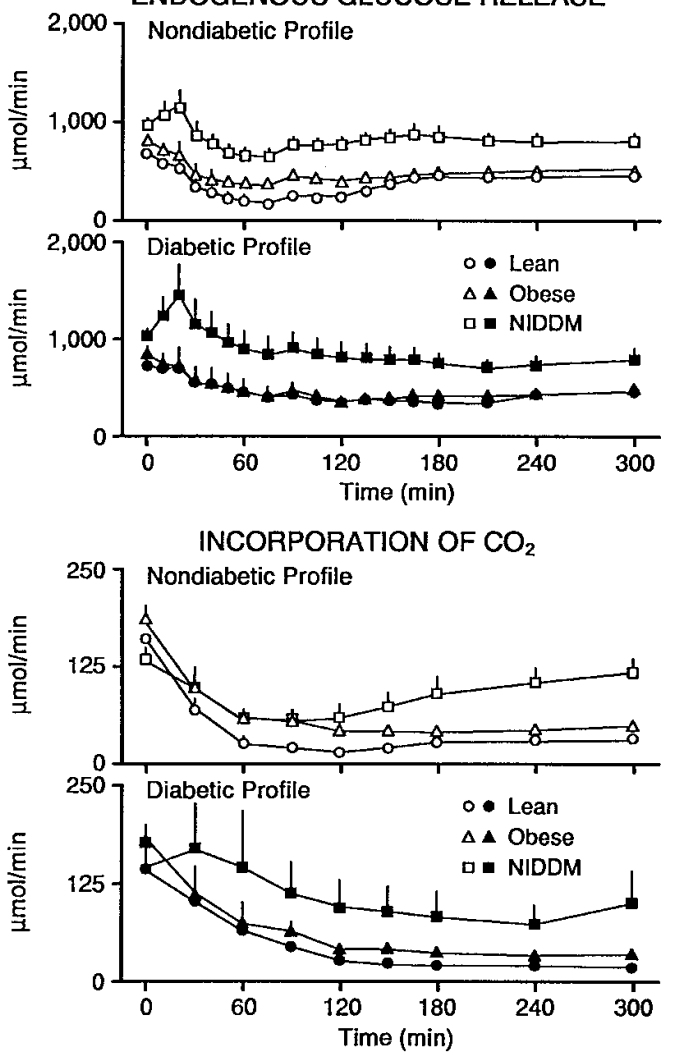

GLUCOSE DISAPPEARANCE

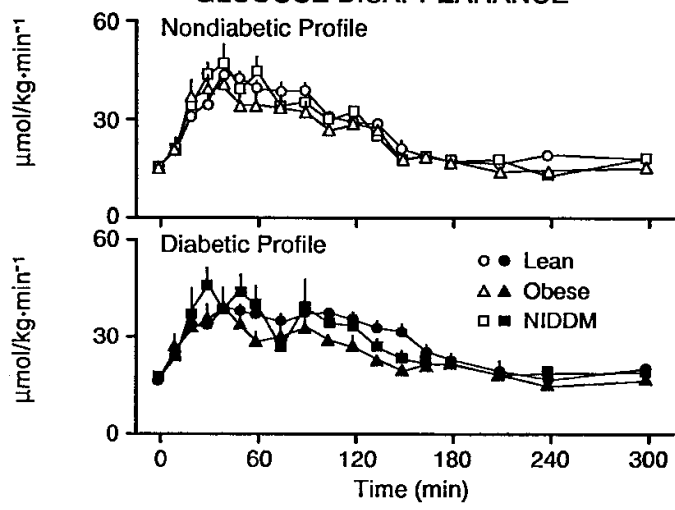

GLUCOSE CLEARANCE

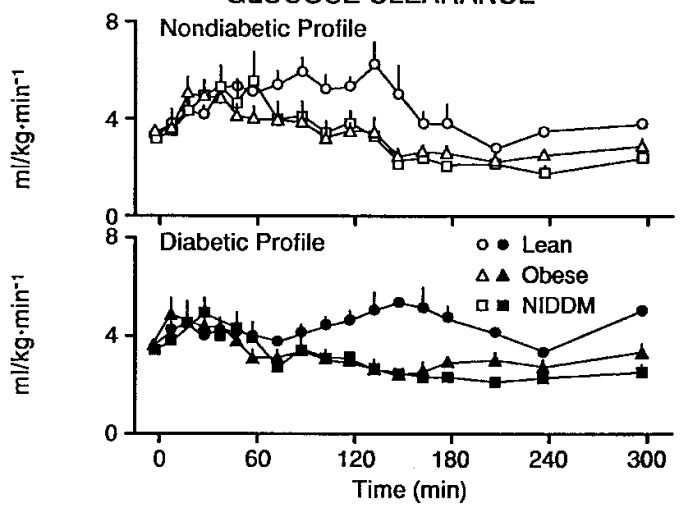

Figure 4. Rates of endogenous glucose release (top left), incorporation of carbon dioxide into glucose (bottom left), glucose disappearance (top right), and glucose clearance (bottom right) observed in lean nondiabetic, obese nondiabetic, and subjects with NIDDM during the nondiabetic and diabetic insulin profiles. 

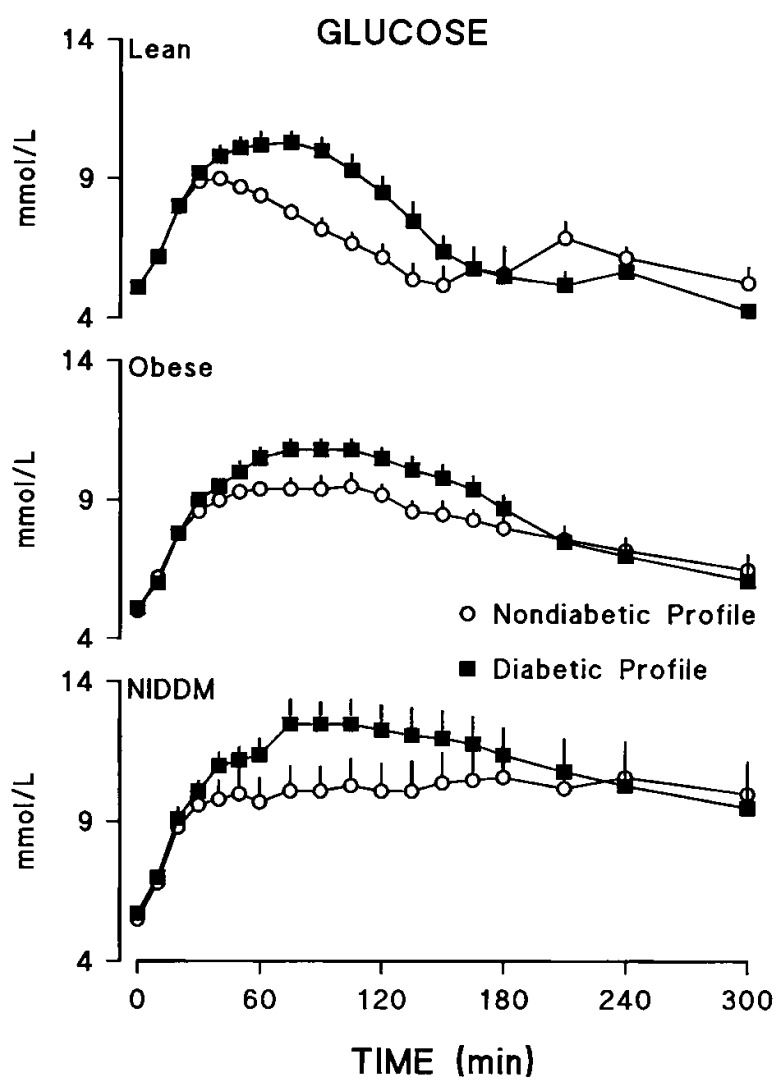
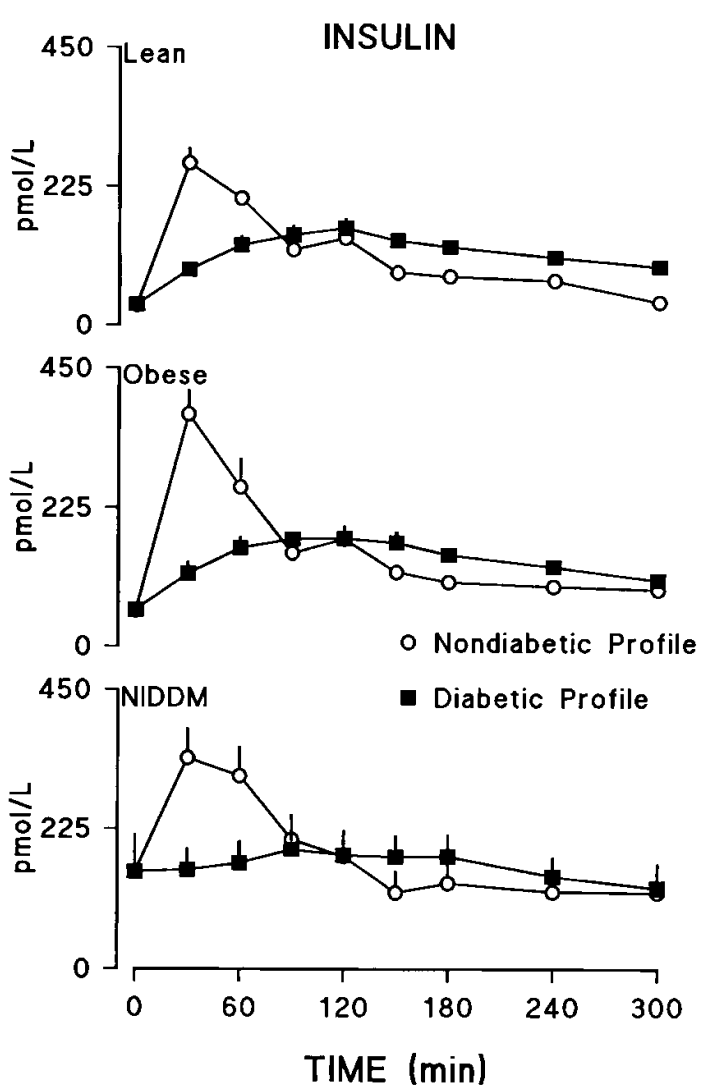

Figure 5. Glucose (left) and insulin (right) concentrations observed in lean nondiabetic, obese nondiabetic, and subjects with NIDDM. A prandial glucose infusion was started at time zero and insulin was administered so as to reproduce a nondiabetic or diabetic insulin profile.

from the fact that glucose clearance was lower $(P<0.05)$ during both insulin profiles compared to that observed in the lean nondiabetic subjects (Fig. 4, bottom right). Glucose clearance did not differ in the obese and NIDDM subjects whether calculated before or after correction for urinary glucose losses. However, a greater $(P<0.05)$ portion of glucose disappearance was accounted for by urinary glucose excretion in the NIDDM than by obese subjects during both the nondiabetic $(346 \pm 185 \mathrm{vs} 11 \pm 5 \mathrm{mmol} / \mathrm{kg}$ per $5 \mathrm{~h}$ ) and diabetic (405 $\pm 154 \mathrm{vs}$ $21 \pm 8 \mathrm{mmol} / \mathrm{kg}$ per $5 \mathrm{~h}$ ) insulin profiles.

\section{Effects of alterations in the pattern of insulin secretion on glucose tolerance}

Glucose and insulin concentrations during the nondiabetic and diabetic insulin profiles. To determine the effects of differences in the pattern of insulin secretion on glucose tolerance in individuals with varying degrees of insulin resistance, results observed in the lean nondiabetic, obese nondiabetic, and obese NIDDM subjects during the nondiabetic insulin profile were compared to those observed in the same individuals when they received an identical amount of insulin in the form of a diabetic insulin profile.

Basal insulin concentrations were the same on both study days in all groups (Fig. 5). The diabetic and nondiabetic insulin profiles produced a pattern of change in insulin concentrations in all three groups that closely mimicked those observed after mixed meal ingestion (compare Fig. 5 to Fig. 1). The nondiabetic insulin profile resulted in a more rapid increase in plasma insulin concentrations than did the diabetic insulin profile in all groups (Fig. 5, right). Insulin concentrations were greater $(P<0.02)$ during the first $2 \mathrm{~h}$ of the nondiabetic than the diabetic insulin profiles in all groups. On the other hand, since identical amounts of insulin were infused in all subjects, integrated insulin concentrations over the $5 \mathrm{~h}$ of study did not differ during the diabetic and nondiabetic profiles in any group.

Basal glucose concentrations were the same on both study days in all three groups. During the first $2 \mathrm{~h}$ of the prandial glucose infusion, plasma glucose concentration rose more (Fig. 5, left) in the presence of the diabetic than nondiabetic insulin profile, resulting in a higher $(P<0.02)$ peak in the lean nondiabetic (10.6 \pm 0.4 vs $9.0 \pm 0.2 \mathrm{pmol} /$ liter) and obese nondiabetic (11.1 $\pm 0.4 \mathrm{vs} 9.8 \pm 0.4 \mathrm{pmol} / \mathrm{liter})$ subjects as well as in the people with NIDDM $(12.9 \pm 0.9$ vs $10.9 \pm 0.7$ pmol/liter $)$. The difference in glucose concentrations, however, was only transient since glucose concentrations by the end of the diabetic insulin profile no longer differed from those observed during the nondiabetic profile. The net effect of these changes was that the integrated glycemic response above basal over the entire $5 \mathrm{~h}$ of study was only slightly greater during the diabetic than the nondiabetic profile (Fig. 4), with the difference reaching statistical significance $(P<0.01)$ in the lean subjects and being of borderline significance in the obese nondiabetic $(P=0.05)$ and $\operatorname{NIDDM}(P=0.06)$ subjects. Of interest, the difference between the integrated glycemic response observed during the nondiabetic and diabetic insulin profiles did not differ in the lean, obese, and NIDDM groups (diabetic minus nondiabetic areas in Table III).

Glucose turnover during the nondiabetic and diabetic insulin profiles. The nondiabetic and diabetic insulin profiles in- 

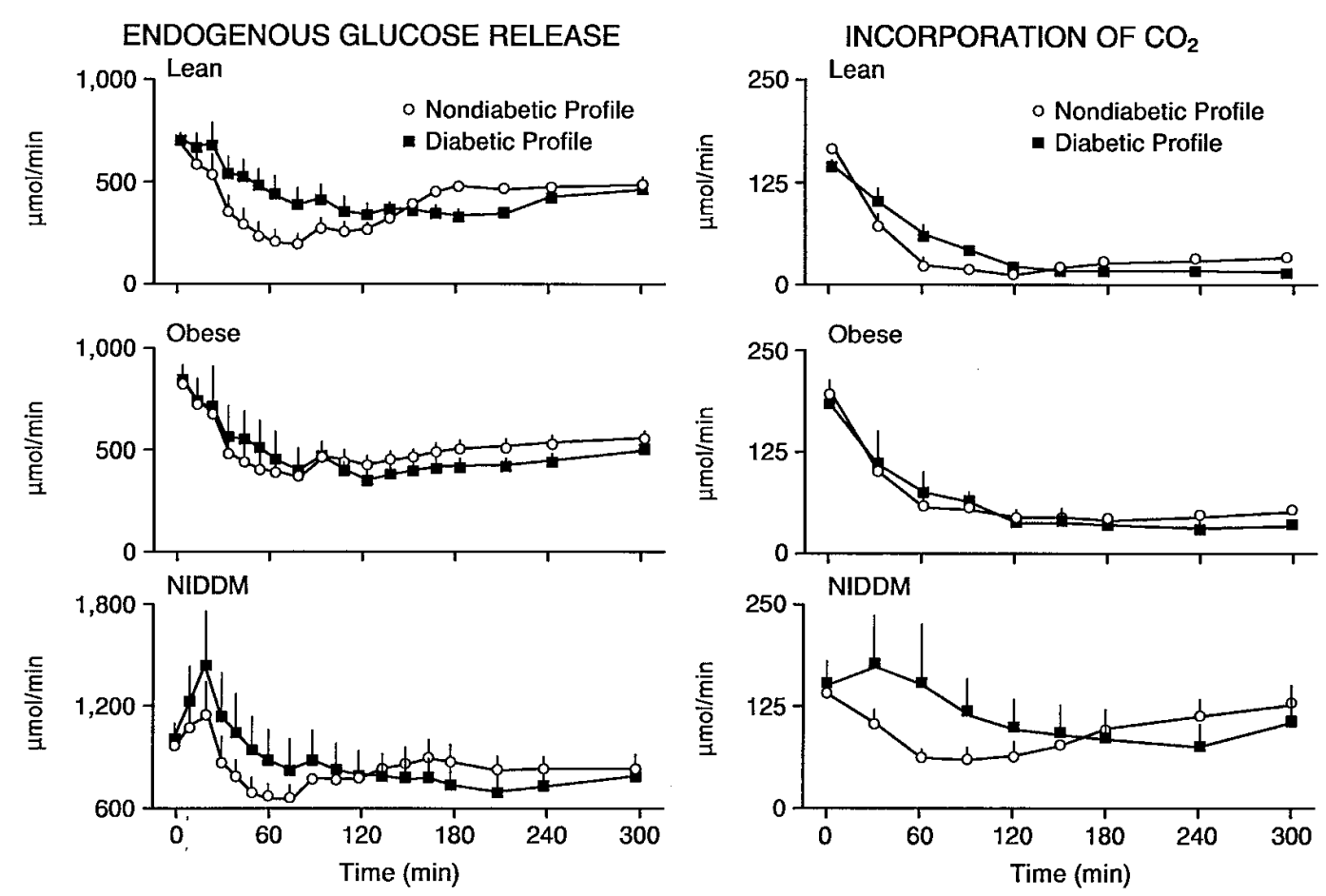

Figure 6. Rates of endogenous glucose release (left) and incorporation of carbon dioxide into glucose (right) observed in lean nondiabetic, obese nondiabetic, and subjects with NIDDM during the nondiabetic and diabetic insulin profiles.

hibited endogenous glucose release in all groups (Fig. 6, left). The pattern of change in endogenous glucose release mirrored that of insulin; endogenous glucose release fell as insulin concentrations rose and rose as insulin concentrations fell. The more rapid increase in insulin during the nondiabetic than dia- betic insulin profile resulted in more rapid $(P<0.05)$ suppression of both endogenous glucose release and the rate of incorporation of carbon dioxide into glucose (an index of gluconeogenesis) in the lean nondiabetic subjects during the first $2 \mathrm{~h}$ of study, with rates merging thereafter (Fig. 6). Sup-
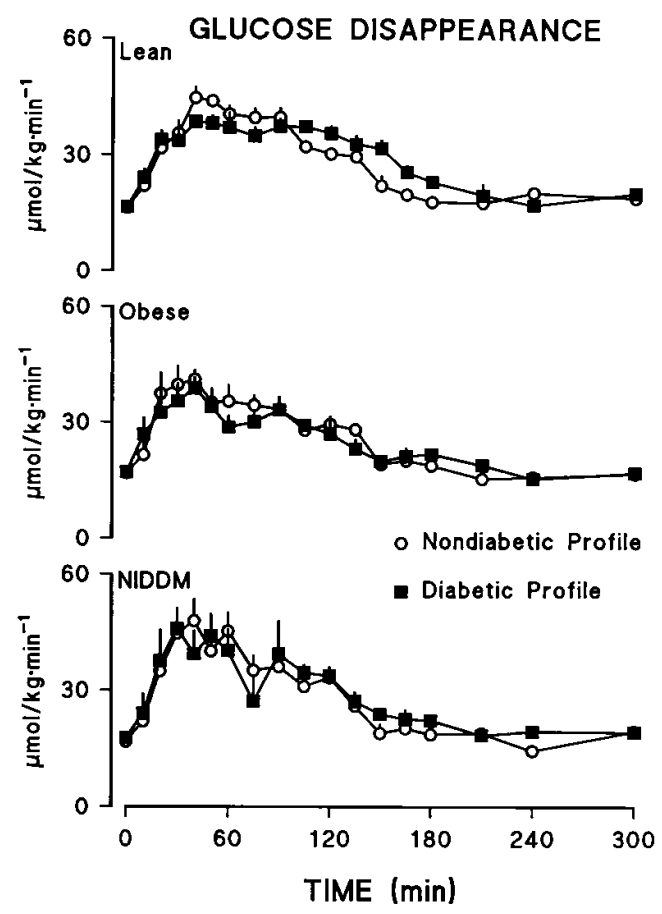
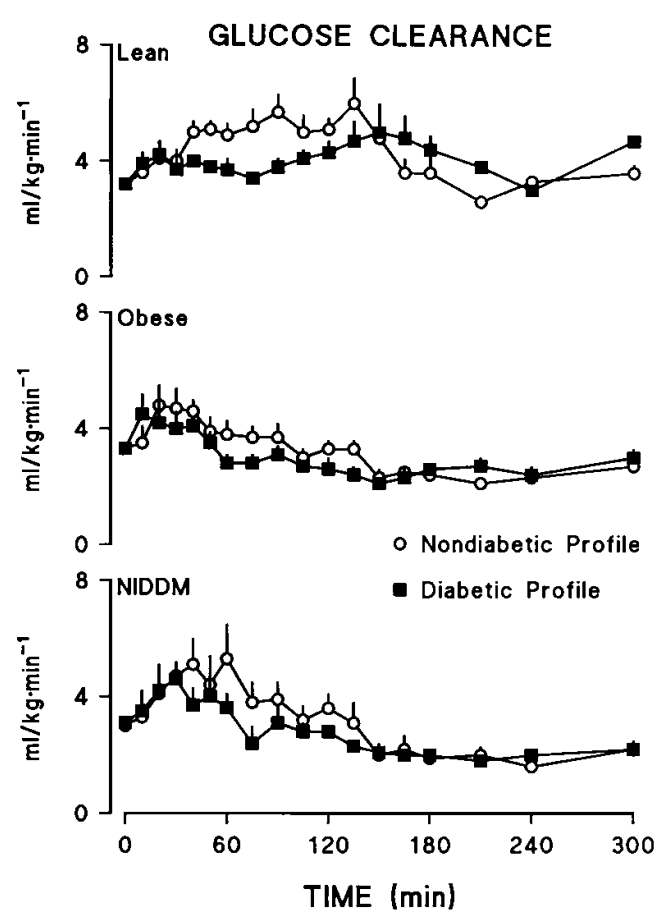

Figure 7. Rates of glucose disappearance (left) and glucose clearance (right) observed in lean nondiabetic, obese nondiabetic, and subjects with NIDDM during the nondiabetic and diabetic insulin profiles. 
pression also tended to be more rapid during the nondiabetic than the diabetic insulin profiles in the other two groups; however, the variation was greater and the differences were not statistically significant.

Glucose disappearance increased to a similar extent in all groups (Fig. 7, left). However, disposal was not appropriate for the higher prevailing glucose concentrations in either of the nondiabetic groups since glucose clearance over the first $2 \mathrm{~h}$ of study was lower $(P<0.05)$ during the diabetic than nondiabetic insulin profile (Fig. 7, right). A similar, but statistically nonsignificant pattern was observed in the NIDDM subjects. As with endogenous glucose release, differences in clearance were only evident during the first $2 \mathrm{~h}$ when the differences in insulin concentrations were the greatest.

\section{Discussion}

While it is well established that people with NIDDM have defects in both insulin secretion and action (1-14), the relative contribution of each to altered glucose tolerance remains unclear. Under conditions of daily living, a change in insulin action results in a change in glucose concentration which in turn causes a change in insulin secretion. Methods such as euglycemic and hyperglycemic clamps circumvent this feedback loop by assessing insulin action and/or insulin secretion in the presence of fixed, constant glucose concentrations. While these approaches allow measurement of the severity of defects in secretion and action, they do not permit determination of the impact of these defects on glucose tolerance. This is not a trivial problem since hyperglycemia can compensate for insulin lack and the rate change of glucose production and utilization in response to a change in insulin may be more important than the steady state response assessed by traditional glucose clamps. Therefore, isolated alterations in the pattern of insulin secretion and action may have different qualitative as well as quantitative effects on glucose tolerance. Furthermore, defects in both may be synergistic or additive.

The present studies sought to address these questions by using an experimental paradigm that evaluated the impact of defects in the pattern of insulin secretion or action by creating nondiabetic and diabetic insulin profiles in individuals who had differing degrees of insulin resistance, namely, lean nondiabetic, obese nondiabetic, and obese NIDDM subjects. Although insulin secretion varies from individual to individual, the pattern of change in postprandial insulin concentrations is remarkably consistent by the time frank hyperglycemia develops (1-9). In the present studies, we fed a group of individuals with NIDDM a standard mixed meal, and then used the observed insulin concentrations to create a typical diabetic insulin profile. The temporal pattern of this profile, namely, a blunted and delayed peak with a total integrated response that did not differ from that in nondiabetic subjects fed the same meal, is virtually identical to that which we and other investigators have observed in other NIDDM subjects following food ingestion (1-9). Thus, while there is no such thing as a single postprandial diabetic insulin profile, the one employed in the present experiments closely mimics that observed in a large number of people with NIDDM.

Previous studies have established the importance of the timing of insulin secretion on glucose tolerance (30-34). Consistent with the present results, those studies demonstrated that, for any given glucose challenge, a delay in insulin delivery results in a greater increment in plasma glucose concentration (30-34). The present experiments extend those studies by examining the effects of alterations in the pattern of insulin secretion in the presence and absence of insulin resistance under conditions simulating those observed after eating. Each subject received $\sim 35 \mathrm{~g}$ of glucose, mimicking the amount of glucose that normally enters the systemic circulation after ingestion of $50 \mathrm{~g}$ of carbohydrate $(4,6,8)$. Each subject also was infused with insulin. When given so as to reproduce a nondiabetic profile, it was sufficient to result in glucose concentrations in lean insulin-sensitive individuals that were virtually superimposable upon those observed in the nondiabetic subjects following ingestion of $50 \mathrm{~g}$ of carbohydrate. Administration of an identical amount of insulin to the lean nondiabetic subjects as a diabetic insulin profile, resulted in an $\sim 1.5 \mathrm{mmol} / \mathrm{liter}$ $(\sim 25 \mathrm{mg} / \mathrm{dl})$ greater increment in glucose concentration. However, glucose concentrations rapidly fell back to levels present during the nondiabetic profile. Despite wide differences in insulin action (see below), the pattern was essentially the same in all three groups. These data indicate that an isolated defect in the pattern of insulin secretion of a magnitude typically observed in most frankly hyperglycemic NIDDM subjects in itself causes only modest and transient hyperglycemia in lean nondiabetic individuals.

Inspection of the glucose turnover data provides insight as to why the differences in glucose concentrations during the nondiabetic and diabetic insulin profiles were not sustained. Both insulin and glucose interact to regulate glucose production and disposal (14-20). An increase in either glucose or insulin suppresses endogenous glucose release and stimulates glucose uptake; a concurrent rise in both glucose and insulin exerts a greater effect than a rise in either alone. The more rapid increase in insulin during the first portion of the nondiabetic insulin profile resulted in a more rapid suppression of endogenous glucose release. The concomitant decrease in the rate of incorporation of carbon dioxide into glucose suggests that the fall in endogenous glucose release was, at least in part, due to inhibition of gluconeogenesis $(6,33,34)$. Differences were most evident in the lean subjects. However, the pattern of production and disposal changed with time; glucose concentrations were higher during the diabetic insulin profile from $1 \mathrm{~h}$ onward. This further suppressed glucose release and further increased glucose disappearance. Although rates of disappearance were not appropriate for the prevailing glucose concentration (i.e., glucose clearance was decreased), the net effect of these changes was the return of glucose concentrations to levels observed during the nondiabetic profile. Thus, the higher late glucose and insulin concentrations present during the diabetic profile compensated for the early delay in the pattern of insulin secretion, thereby minimizing the overall effect on glucose tolerance. It is important to point out, however, that the present experiments only studied the effect of an alteration in the timing of insulin delivery since the amounts of insulin infused were identical on both occasions. A combined delay and reduction in insulin response is likely to cause more marked hyperglycemia than either one alone.

Since all subjects were given identical amounts of insulin and glucose, the glycemic excursion was determined by the interaction between insulin action and glucose effectiveness (defined as the ability of glucose to stimulate its own uptake and to suppress its own release). We have previously demonstrated in a separate set of experiments that glucose effectiveness in 
presence of the same nondiabetic insulin profile does not differ in NIDDM and nondiabetic subjects (20). Therefore, the greater rise in glucose concentrations in the NIDDM subjects during the nondiabetic insulin profile was solely caused by insulin resistance. We are unaware of any studies comparing glucose effectiveness in lean and obese nondiabetic subjects in the presence of the glucose and insulin concentrations resembling those present in the current experiments. If glucose effectiveness is impaired by obesity per se, then the higher glucose concentrations observed in the obese than in the lean nondiabetic subjects may have been caused in part by factors other than insulin resistance.

A defect in insulin action had a different temporal effect on glucose tolerance than did a decrease in the pattern of insulin secretion. A decrease in insulin action only minimally influenced peak glucose concentration, but markedly prolonged the duration of hyperglycemia. A decrease in insulin action to a level observed in people with NIDDM had a much greater impact on glucose tolerance than did a decrease in the pattern of insulin secretion. Whereas a decrease early in the pattern of insulin secretion led to only a slight increase in the glucose area above basal (compare glucose areas during nondiabetic and diabetic profiles in Table III), a decrease in insulin action to a level caused by obesity alone led to a 2.5 -fold increase in the glucose area above basal (compare glucose areas during nondiabetic profiles in lean and obese subjects in Table III). Even more impressive, a decrease in insulin action to a level caused by obesity plus NIDDM led to a 4.2-fold greater glycemic excursion (compare glucose areas during nondiabetic profiles in lean and NIDDM subjects in Table III). Concurrent defects in the pattern of insulin secretion and action caused a greater deterioration in glucose tolerance than either alone. Thus, in contrast to a decrease in the pattern of insulin secretion, insulin resistance caused sustained and marked hyperglycemia.

The ability of insulin to suppress endogenous glucose release and to stimulate glucose disposal differed depending on the degree of insulin resistance. Whereas hyperglycemia in the obese nondiabetic subjects was associated with impaired glucose clearance, hyperglycemia in the more insulin-resistant NIDDM subjects was due to both decreased glucose clearance and excessive endogenous glucose release. Although the degree of impairment in glucose clearance was similar in the NIDDM and obese nondiabetic subjects, urinary glucose loss was greater in the diabetic subjects, implying lower tissue glucose uptake. In addition, glucose concentrations remained essentially flat in the NIDDM subjects during the last few hours of the study, at which time tissue glucose uptake and renal glucose excretion equaled the (elevated) rate of endogenous glucose release. A similar situation occurred in the obese individuals. However, since endogenous glucose release was lower, this equilibrium was achieved at lower glucose concentrations, with urinary glucose excretion making a negligible contribution. Thus the greater the defect in insulin action, the higher the glucose concentration had to rise to balance glucose appearance and disappearance. Of note, as is evident from Fig. 3, the addition of hepatic insulin resistance to an inappropriately low rate of glucose disappearance was sufficient to convert the modestly elevated glucose concentrations in the nondiabetic obese subjects to frankly diabetic levels (35) in the NIDDM subjects. This occurred despite the fact that the latter, if anything, had a lower percentage of body fat than the former, im- plying that factors other than obesity contributed to insulin resistance in the NIDDM subjects (Table I).

Limitations. As with all studies, the present experiments have limitations. Ideally, insulin should have been infused into the portal venous system. However, recent experiments have demonstrated that peripheral infusion of insulin has an equal, if not greater, effect than does portal infusion (36-38). Since insulin was infused in the same manner in all subjects, the route of insulin delivery is unlikely to have influenced our conclusions. Endogenous insulin secretion was inhibited and the same amount of insulin was infused in all subjects. However, since basal insulin concentrations were higher, insulin concentrations during the insulin profiles also tended to be higher in the NIDDM subjects. This, if anything, would lead to an underestimate of the severity of insulin resistance. We do not know what the portal glucagon concentrations actually were during the experiments. However, assuming that portal venous glucagon concentrations were $40 \%$ higher than peripheral glucagon concentrations before somatostatin infusion, but approximated peripheral glucagon concentrations during somatostatin infusion (39-42), portal glucagon concentrations in all groups fell from $\sim 140 \mathrm{pg} / \mathrm{ml}$ at time zero to $\sim 100 \mathrm{pg} / \mathrm{ml}$ from 30 to $120 \mathrm{~min}$, and then rose back to $\sim 140 \mathrm{pg} / \mathrm{ml}$ from 120 to 300 min (Fig. 2). This pattern of suppression, which mimics that observed in nondiabetic subjects after food ingestion, does not normally occur in people with diabetes mellitus (5-9). Therefore, defects in insulin secretion and/or action of a magnitude similar to those present in the current experiments may lead to even greater hyperglycemia when postprandial suppression of glucagon is impaired (43).

Glucose was infused rather than ingested. This approach was used since somatostatin is a potent inhibitor of gastric emptying (44), and somatostatin was necessary to ensure that endogenous insulin secretion was equally inhibited in all groups. Intravenous infusion of glucose also avoids the uncertainties introduced by variations in intestinal absorption. However, this precludes assessment of the effects of differences in insulin secretion/action on first pass splanchnic glucose uptake. Future studies will be required to address this question. Measurement of the rate of incorporation of carbon dioxide into glucose provides a qualitative rather than quantitative estimate of gluconeogenesis $(6,45,46)$. The limitations of this, as well as other similar isotopic approaches for the measurement of gluconeogenesis in vivo, have been previously discussed in detail $(6,45,46)$.

The method used to assess insulin action is a modification of the so-called insulin suppression test $(10,11)$. Insulin was given as a continuously changing infusion not only because this afforded us the opportunity to compare nondiabetic and insulin diabetic profiles, but also because insulin administered in this fashion has a greater biologic effect than the identical amount of insulin given as a constant infusion (47). Glucose concentrations were maintained in the euglycemic range in the NIDDM subjects during the night. This was done so that glucose concentrations would begin at the same level in all groups. If normalization of glucose concentration during the night improved insulin action in the NIDDM subjects, then we may have underestimated the impact of insulin resistance in these subjects. However, this would be a conservative error since, as it was, the effect of insulin resistance on carbohydrate tolerance was dramatic.

Conclusions. The present experiments demonstrate that 
an isolated defect in insulin secretion or action of a magnitude typical of that present in people with NIDDM causes hyperglycemia. However, the severity and pattern of hyperglycemia differs. A delay in insulin secretion is associated with transiently higher peak glucose concentrations, resulting in a modest increase in the integrated glycemic response. In contrast, defects in insulin action markedly impair glucose tolerance by prolonging the duration of hyperglycemia. Both cause hyperglycemia by altering the pattern and/or rate of change of endogenous glucose release and the efficiency of glucose uptake. Whereas obese nondiabetic and NIDDM subjects had comparable defects in glucose clearance, the addition of hepatic insulin resistance in the latter converted a glucose profile that was glucose intolerant according to National Diabetes Data Group criteria to frank diabetes mellitus (35). If avoidance of hyperinsulinemia is desirable, these data suggest that agents that enhance insulin action are likely to result in a greater improvement in glucose tolerance in people with NIDDM than are agents that act solely by restoring early postprandial insulin secretion to normal. They also suggest that such agents will need to increase both hepatic and extrahepatic response to insulin if postprandial glucose metabolism is to be completely restored to normal.

\section{Acknowledgments}

We thank T. Madson and D. Nash for their technical assistance, A. Wagner for assistance in preparation of the manuscript, and the staff of the Clinical Research Center for assistance in performing the studies.

This work was supported by U.S. Public Health Service (DK29953, RR00585), the Mayo Foundation, and Ministero Universita Ricerca Scientifica Tecnica, Italy. Dr. A. Basu was supported by a mentor-based American Diabetes Association Research Fellowship Award. Dr. S. Dinneen received funding from the Endocrine Fellows Foundation.

\section{References}

1. Bantle, J.P., D.C. Laine, G.W. Castle, J.W. Thomas, B.J. Hoogwerf, and F.C. Goetz. 1983. Postprandial glucose and insulin responses to meals containing different carbohydrates in normal and diabetic subjects. N. Engl. J. Med. 309:7-12.

2. Reaven, G.M., C.B. Hollenbeck, and Y.-D.I. Chen. 1993. Relationship between glucose tolerance, insulin secretion, and insulin action in non-obese individuals with varying degrees of glucose tolerance. Diabetologia. 32:52-55.

3. Osei, K., J.M. Falko, T.M. O'Dorisio, P.G. Fields, and B. Bossetti. 1986. Gastric inhibitory polypeptide responses and glucose turnover rates after natural meals in type II diabetic patients. J. Clin. Endocrinol. \& Metab. 62:325-330.

4. Firth, R.G., P.M. Bell, H.M. Marsh, I. Hansen, and R.A. Rizza. 1986. Postprandial hyperglycemia in patients with noninsulin-dependent diabetes mellitus: role of hepatic and extrahepatic tissues. J. Clin. Invest. 77:1525-1532.

5. Ferrannini, E., D.C. Simonson, L.D. Katz, G. Reichard, Jr., S. Bevilacqua, E.J. Barrett, M. Olsson, and R.A. DeFronzo. 1988. The disposal of an oral glucose load in patients with non-insulin-dependent diabetes. Metabolism. 37: 79-85.

6. McMahon, M., H.M. Marsh, and R.A. Rizza. 1989. Effects of basal insulin supplementation on disposition of mixed meal in obese patients with NIDDM. Diabetes. 38:291-303.

7. Mitrakou, A., D. Kelley, T. Veneman, T. Jenssen, T. Pangburn, J. Reilly, and J. Gerich. 1990. Contribution of abnormal muscle and liver glucose metabolism to postprandial hyperglycemia in NIDDM. Diabetes. 39:1381-1390.

8. Butler, R.C., and R.A. Rizza. 1991. Contribution to postprandial hyperglycemia and effect on initial splanchnic glucose clearance of hepatic glucose cycling in glucose-intolerant or NIDDM patients. Diabetes. 40:73-81.

9. Kelley, D., M. Mokan, and T. Veneman. 1994. Impaired postprandial glucose utilization in non-insulin-dependent diabetes mellitus. Metabolism. 43: 1549-1557.

10. Shen, S.W., G.M. Reaven, and J.W. Farquhar. 1970. Comparison of impedance to insulin-mediated glucose uptake in normal subjects and in subjects with latent diabetes. J. Clin. Invest. 49:2151-2160.
11. Harano, Y., S. Ohgaku, H. Hidaka, K. Haneda, R. Kikkawa, Y. Shigeta, and H. Abe. 1977. Glucose, insulin and somatostatin infusion for the determination of insulin sensitivity. J. Clin. Endocrinol. \& Metab. 45:1124-1127.

12. DeFronzo, R., D. Deibert, R. Hendler, P. Felig, and V. Soman. 1979. Insulin sensitivity and insulin binding to monocytes in maturity-onset diabetes. $J$. Clin. Invest. 63:939-946.

13. Kolterman, O.G., R.S. Gray, J. Griffin, P. Burstein, J. Insel, J.A. Scarlett, and J.M. Olefsky. 1981. Receptor and postreceptor defects contribute to the insulin resistance in noninsulin-dependent diabetes mellitus. J. Clin. Invest. 68:957-969.

14. Rizza, R.A., L.J. Mandarino, and J.E. Gerich. 1981. Mechanism and significance of insulin resistance in non-insulin-dependent diabetes mellitus. Diabetes. 30:990-995.

15. Cherrington, A.D., P.E. Williams, and M.S. Harris. 1978. Relationship between the plasma glucose level and glucose uptake in the conscious dog. $\mathrm{Me}$ tabolism. 27:787-791.

16. Sacca, L., R. Hendler, and R.S. Sherwin. 1978. Hyperglycemia inhibits glucose production in man independent of changes in glucoregulatory hormones. J. Clin. Endocrinol. \& Metab. 47:1160-1161.

17. Best, J.D., G.J. Taborsky, Jr., J.B. Halter, and D. Porte, Jr. 1981. Glucose disposal is not proportional to plasma glucose level in man. Diabetes. 30: 847-850.

18. Hansen, I.L., P.E. Cryer, and R.A. Rizza. 1985. Comparison of insulinmediated and glucose-mediated glucose disposal in patients with insulin-dependent diabetes mellitus and in nondiabetic subjects. Diabetes. 34:751-755.

19. Capaldo, B., D. Santoro, G. Riccardi, N. Perrotti, and L. Saccà. 1986. Direct evidence for a stimulatory effect of hyperglycemia per se on peripheral glucose disposal in type II diabetes. J. Clin. Invest. 77:1285-1290.

20. Alzaid, A.A., S.F. Dinneen, D.J. Turk, A. Caumo, C. Cobelli, and R.A. Rizza. 1994. Assessment of insulin action and glucose effectiveness in diabetic and nondiabetic humans. J. Clin. Invest. 94:2341-2348.

21. Nolan, J.J., B. Ludvik, P. Beerdsen, M. Joyce, and J. Olefsky. 1994. Improvement in glucose tolerance and insulin resistance in obese subjects treated with troglitazone. N. Engl. J. Med. 331:1188-1193.

22. Gutniak, M., C. Orskov, J.J. Holst, B. Ahren, and S. Efendic. 1992. Antidiabetogenic effect of glucagon-like peptide-1 (7-36)amide in normal subjects and patients with diabetes mellitus. N. Engl. J. Med. 326:1316-1322.

23. Howey, D.C., R.R. Bowsher, R.L. Brunelle, and J.R. Woodworth. 1994. [Lys(B28), Pro(B29)]-human insulin. A rapidly absorbed analogue of human insulin. Diabetes. 43:396-402.

24. Shuster, L.T., V.L.W. Go, R.A. Rizza, P.C. O'Brien, and F.J. Service. 1988. Incretin effect due to increased secretion and decreased clearance of insulin in normal humans. Diabetes. 37:200-203.

25. White, N.H., D. Skor, and J.V. Santiago. 1982. Practical closed-loop insulin delivery. A system for the maintenance of overnight euglycemia and the calculation of basal insulin requirements in insulin-dependent diabetics. Ann. Intern. Med. 97:210-213.

26. Katz, H., M. Homan, M. Jensen, A. Caumo, C. Cobelli, and R. Rizza. 1994. Assessment of insulin action in NIDDM in the presence of dynamic changes in insulin and glucose concentration. Diabetes. 43:289-296.

27. Bradley, D.C., G.M. Steil, and R.N. Bergman. 1993. Quantitation of measurement error with optimal segments: basis for adaptive time course smoothing. Am. J. Physiol. 264:E902-911.

28. Steele, R., J.S. Wall, R.C. De Bodo, N. Altszuler, S.P. Kiang, and C. Bjerknes. 1956. Measurement of size and turnover rate of body glucose pool by the isotope dilution method. Am. J. Physiol. 187:15-24.

29. Tiku, M.L. 1982. Robust statistics for testing equality of means or variances. Communications in Statistics-Theory and Methods. 11:2543-2558.

30. Kraegen, E.W., D.J. Chisholm, and M.E. McNamara. 1981. Timing of insulin delivery with meals. Horm. Metab. Res. 13:365-367.

31. Bratusch-Marrain, P.R., and W.K. Waldhäusl. 1985. Impact of the "early peak" of insulin secretion on glucose utilization in man. Horm. Metabol. Res. 17:163-164.

32. Bruce, D.G., D.J. Chisholm, L.H. Storlien, and E.W. Kraegen. 1988. Physiological importance of deficiency in early prandial insulin secretion in non-insulin-dependent diabetes. Diabetes. 37:736-744.

33. Luzi, L., and R.A. DeFronzo. 1989. Effect of loss of first-phase insulin secretion on hepatic glucose production and tissue glucose disposal in humans. Am. J. Physiol. 257:E241-E246.

34. Calles-Escandon, J., and D.C. Robbins. 1987. Loss of early phase of insulin release in humans impairs glucose tolerance and blunts thermic effect of glucose. Diabetes. 36:1167-1172.

35. National Diabetes Data Group. 1979. Classification and diagnosis of diabetes mellitus and other categories of glucose intolerance. Diabetes. 28:10391057.

36. Katz, H., M. Homan, J. Velosa, P. Robertson, and R. Rizza. 1991. Effects of pancreas transplantation on postprandial glucose metabolism. N. Engl. J. Med. 325:1278-1283.

37. Giacca, A., S.J. Fisher, Z.Q. Shi, R. Gupta, H.L.A. Lickley, and M. Vranic. 1992. Importance of peripheral insulin levels for insulin-induced suppression of glucose production in depancreatized dogs. J. Clin. Invest. 90:1769-1777.

38. Bradley, D.C., R.A. Poulin, and R.N. Bergman. 1993. Dynamics of he- 
patic and peripheral insulin effects suggest common rate-limiting step in vivo. Diabetes. 42:296-306.

39. Jaspan, J.B., J. Ruddick, and E. Rayfield. 1984. Transhepatic glucagon gradients in man: evidence for glucagon extraction by human liver. J. Clin. Endocrinol. \& Metab. 58:287-292.

40. Blackard, W.G., N.C. Nelson, and S.S. Andrews. 1974. Portal and peripheral vein immunoreactive glucagon concentrations after arginine or glucose infusions. Diabetes. 23:199-202.

41. Jaspan, J.B., A.H. Huen, C.G. Morley, A.R. Moossa, and A.H. Rubenstein. 1977. The role of the liver in glucagon metabolism. J. Clin. Invest. 60:421428 .

42. Jaspan, J.B., K.S. Polonsky, M. Lewis, J. Pensler, W. Pugh, A.R. Moossa, and A.H. Rubenstein. 1981. Hepatic metabolism of glucagon in the dog: contribution of the liver to overall metabolic disposal of glucagon. Am. J.
Physiol. 240:E233-E244.

43. Dinneen, S., A. Alzaid, D. Turk, and R. Rizza. 1995. Failure of glucagon suppression contributes to postprandial hyperglycemia in IDDM. Diabetologia. 38:337-343.

44. Johansson, C., O. Wisen, S. Efendic, and K. Uvnuäs-Wallensten. 1981. Effects of somatostatin on gastrointestinal propagation and absorption of oral glucose in man. Digestion. 22:126-137.

45. Katz, J. 1985. Determination of gluconeogenesis in vivo with ${ }^{14} \mathrm{C}$ labeled substrates. Am. J. Physiol. 17:391-399.

46. Brosnan, J.T. 1982. Pathways of carbon flux in gluconeogenesis. Fed. Proc. 41:91-94.

47. Doeden, B., and R. Rizza. 1987. Use of variable insulin infusion to assess insulin action in obesity: defects in both the kinetics and amplitude of response. J. Clin. Endocrinol. \& Metab. 64:902-908. 